\title{
ResearchOnline@JCU
}

This is the Accepted Version of a paper published in the Scandinavian Journal of Occupational Therapy

Barnett, Fiona A, Che Daud, Ahmad Zamir, Yau, Matthew, and Judd, Jennifer (2016) Occupation based intervention in hand injury rehabilitation:

experiences of occupational therapists in Malaysia. Scandinavian Journal of Occupational Therapy, 23(1). pp. 57-66.

http://dx.doi.org/10.3109/11038128.2015.1062047 


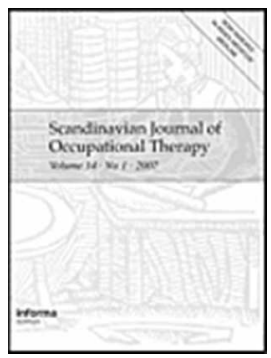

\section{Occupation based intervention in hand injury rehabilitation: Experiences of occupational therapists in Malaysia}

\begin{tabular}{|r|l|}
\hline Journal: & Scandinavian Journal of Occupational Therapy \\
\hline Manuscript ID: & SOcC-2014-0136 \\
\hline Manuscript Type: & Original article \\
\hline Keywords: & $\begin{array}{l}\text { Hand Injuries, Occupational therapy, Occupations, Activities of daily living, } \\
\text { Qualitative research }\end{array}$ \\
\hline \multicolumn{2}{|l}{} \\
\end{tabular}

SCHOLARONE $^{\text {Ix }}$
Manuscripts 


\begin{abstract}
Introduction: Practising Occupation Based Intervention (OBI) uses the concepts of occupation as a means and an end. Occupation as a means refers to occupational and purposeful tasks as a therapeutic agent while occupation as an end refers to occupation as an outcome of intervention. The purpose of this study was to describe the occupational therapists' experiences of providing OBI in hand injury rehabilitation. Methods: Sixteen occupational therapists with more than five years of experience in hand rehabilitation were individually interviewed on their experiences of using OBI in practice. Data was thematically analysed using an Interpretative Phenomenological Analysis. Results: Interpretations of the themes resulted in: “Occupation as a means”, "Occupation as an end", "Benefit of OBI", "Challenges of OBI" and "Making OBI a reality". It was found that occupational therapists had positive experiences with OBI. Occupational therapists perceived that occupation as a means and an end can be merged in a single therapy session when the occupational therapists use an occupation that is therapeutic. Conclusion: Although occupation as a means and an end have different purposes, when the ultimate goal is, to enhance the clients' maximum level of functioning, both can be used for successful rehabilitation of hand injuries.
\end{abstract}

\title{
Keywords
}

Hand Injuries, occupational therapy, occupations, activities of daily living, qualitative research 
OCCUPATION BASED INTERVENTION IN HAND INJURY REHABILITATION

\section{Introduction}

Hand injuries impact on the individual's ability to perform daily occupations. The area of occupation includes Activities of Daily Living (ADLs), Instrumental Activities of Daily Living (IADLs), rest and sleep, education, play, leisure and social participation (1). People with hand injuries may experience various difficulties in daily occupation, depending on the level of severity. Most people with unilateral hand injuries reported that everyday occupations like washing, dressing, cooking and cleaning became more complex and challenging to perform $(2$, 3).

Malaysia is one of the new industrialized market countries in the world where hand injuries are mostly due to industrial and work-related accidents $(4,5)$. Hand injuries among children in Malaysia are caused by domestic injuries (48\%), road traffic accidents (19\%), school activities (15\%) and leisure activities (12\%) (6). Fireworks-related accidents, especially during festive seasons are another cause of hand injuries in Malaysia (7). The main role of occupational therapy in hand rehabilitation is to assist clients to make adjustments in life and to promote recovery through the use of therapeutic occupations (8).

Occupation Based Intervention (OBI) can be described through the concepts of occupation as a means and an end. 'Occupation as a means' refers to occupations and purposeful tasks as a remediation agent to improve a client's physical function $(9,10)$. Engaging clients with hand injuries in purposeful occupations such as making origami helps to improve function of the injured hands (11). Descriptions of 'occupation as a means' is similar to 'occupation based' as recently described by Fisher (12). 'Occupation as an end' refers to the ultimate goal of 
occupational therapy intervention, which is to restore, improve and enable the clients to engage in purposeful occupations (10). The domain of occupational therapy includes client factors, performance patterns (habits, routines, rituals and roles), performance skills and contexts that need to be considered when implementing 'occupation as an end' with clients (1). Occupational therapists need to be client focused and work towards clients' occupational goals (13). 'Occupation as an end' is similar to 'occupation focused' as described by Fisher (12).

OBI benefits the clients and makes occupational therapy unique as the intervention uses occupational and purposeful tasks, as the core business of occupational therapy $(9,14-16)$. A study in adult clients with mental illness found that OBI increases occupational performance and satisfaction (17). OBI is also effective in minimizing the decline in leisure and ADLs engagement (18). A case study on shoulder adhesive capsulitis reported that providing OBI at the early stages helped to reduce pain, increased range and quality of motion, and improved occupational performance (19). Occupational therapists generally believe that OBI is more effective, individualized, and valuable when generalized to client's everyday life (16). Occupational therapists reported that OBI facilitates meaningful therapeutic experiences, functional activity and holism in hand rehabilitation (20).

Despite the benefits of OBI, several challenges limit occupational therapists from using it in practice, and include logistic issues such as time, limited space, and lack of resources and equipment $(20,21)$. For instance, occupational therapy units set up in the medically-oriented model facilities are equipped with component-driven tools rather than functional equipment (9). The dominance of the medically-oriented model in practice settings challenges OBI, as it focuses 
to eliminate impairments rather than to increase function $(9,20,21)$. Within the medical-oriented model setting occupational therapists found it difficult to incorporate occupational tasks such as cooking, craft and other daily living activities (13). Reimbursement issues for OBI were also a challenge because billing for OBI is complex and not straightforward $(20,21)$. The credibility of OBI is also challenged, as some occupational therapists feel that the interventions cannot meet the client's goal, are too frustrating and that there is no concrete evidence to support this practice (20). The aim of this study was to describe the experiences of Malaysian occupational therapists providing OBI using the concept occupation as a means and an end. This descriptive study provides an in-depth understanding of how $\mathrm{OBI}$ intervention is delivered to the client with hand injuries.

\section{Material and Methods}

\section{Design}

This study used an Interpretative Phenomenology Analysis (IPA) approach. IPA is described as a dynamic process, where the researcher uses an interpretative method to understand the participant's personal world (22). IPA involves a double hermeneutic, of which both participants and researchers are trying to make sense of certain phenomenon or experiences (23). This approach was used to develop a thick description about the participants' experience in using OBI for clients with hand injuries (24). Approvals to conduct this study were obtained from the Human Ethics Committee of James Cook University, Australia (H4558), Institute for Public Health, Malaysia (NMRR-12-53-10918) and Economic Planning Unit, Malaysia (EPU 40/200/19/2865) in accordance with standard ethical guidelines. To adhere to confidentiality and ethical guidelines, all names within the participant quotes are pseudonyms. 


\section{Participants}

Purposive sampling was used to recruit occupational therapists working in hand rehabilitation, with at least five years' experience in this field. Occupational therapists with more than five years' experience in hand rehabilitation in a previous study were considered as experts (25). A list of occupational therapists who met the inclusion criteria was obtained from the head of Malaysia Occupational Therapy Service were invited to participate in the study. Sixteen occupational therapists consented to participate and were interviewed individually. They were qualified with diploma $(n=8,50 \%)$ and bachelor degree $(n=8,50 \%)$ in occupational therapy. A diploma or bachelor degree from the Ministry of Education accredited institutions is required to be an occupational therapist in Malaysia. The demographic details of the participants are shown in the Table 1.

\section{Procedure}

Semi-structured in-depth interviews were used as they allow the researcher to follow up interesting issues as they emerged during the interview (23). All interviews were audio-tape recorded and immediately following each interview, the participant's overall responses were recorded in the researcher's reflective diaries. Interviews were conducted in a quiet room at the occupational therapy unit when the participants were free from any patient or duties. All interviews were conducted by the first author from December 2012 to January 2013. An interview guide was used to obtain rich and comprehensive responses from the participants (26). Some questions from the interview guide were constructed based on literature and the rest of the questions were prepared according to the research objectives. The participants were asked how 
they apply OBI according to the concept of occupation as a means and an end. A brief explanation about the occupation as a means and an end was given to participants who were not familiar with the terms before the interview. Probe and prompt questions were used accordingly during the interview to enrich and reconfirm certain information from and with the participants (26). The interviews lasted between 45-120 minutes depending on the participants' responses. All interviews were transcribed verbatim and checked for accuracy before the analysis.

Data was thematically analysed using the IPA approach as described by Smith (27). The first transcript was read and reread a few times to gain a deep understanding about the participants' personal view. Initial notes were made on the manuscript to develop emergent themes. Connections between the themes were searched to develop super-ordinate themes. Next interviews were analysed and potential or shared themes across the cases were identified to develop final super-ordinate themes and subordinate themes.

\section{Triangulation strategies}

Member checking using a focus group discussion was conducted with four participants to verify the results of the initial analysis, and who were also asked to give comments and add information to clarify the issues further. The comments and additional information from the focus group discussion were then used to strengthen and refine the themes. Triangulation of analysts was employed, where the first and second author did individual coding and then compared results to eliminate potential bias and illuminate blind spots that may arise from a single analyst (26). Indepth and thick descriptions were used to explain the results from the participants' context. 


\section{Results}

Five super-ordinate themes emerged from the analysis, namely "Occupation as a means", “Occupation as an end", "Benefit of OBI", "Challenges of OBI", and "Making OBI a reality". All participants expressed positive experiences despite struggling to provide OBI in hand injury rehabilitation.

\section{Occupation as a means}

This theme illustrates how occupational therapists use occupations and purposeful tasks as the healing agent. Four subordinate themes emerged; treatment modalities, therapeutic benefits, home-based hand therapy and evaluation of hand function. Participants described that the modalities for 'occupation as a means' can be occupations or purposeful tasks. ADLs, AIDLs and leisure are often chosen as treatment modalities. Occupational therapists felt that when providing 'occupation as a means', the occupations and purposeful tasks selected should be relevant and meaningful to the clients. One of the participants said, "I usually provide them with tasks that simulate their [clients] daily occupations" [Hussain]. Participants described that they have to complete the client's occupational profile (e.g. occupational history and experiences, daily living patterns, values, needs and interests) to determine what is meaningful and relevant to the clients.

Before prescribing 'occupation as a means' to the clients, the therapeutic benefits of occupations or purposeful tasks must be properly analysed for better treatment outcomes. The clients were encouraged to use their injured hand as much as possible. "The reason why I encourage my clients to use the injured hand as much as possible is to avoid 'learned non-use' syndrome. 
OCCUPATION BASED INTERVENTION IN HAND INJURY REHABILITATION

Learned non-use occurs when the clients lose their hand functions as a result of not using it"

[Damia]. Participants suggested that challenging tasks with the clients had to have the prospect of success to avoid frustration. The tasks can be graded according to time, level of difficulty, number of repetitions, distance and resistance. Participants used 'occupation as a means' when the clients were over the protective stage and not under any specific treatment protocol such as tendon or nerve repair. "Protective stage is a phase where the injured bone, muscle, joint, nerve or tendon is immobilized for a certain time duration to promote healing and to avoid further damage to the injured structures" [Chan].

Participant Kesuma described that, "One of my clients had poor pinch grip strength after a scaphoid fracture, and I used pinching pegs task to improve the pinch strength. I also advised the client to continue practising with the clothes pegs while she was doing the laundry at home". Other than using 'occupation as a means' in the clinic, the clients were also encouraged to use 'occupation as a means' as a home-based hand therapy. Participants also described that the material and resources are available in the clients' home. "I always think whatever objects or materials the client has at home. We [occupational therapists] can use it as a therapeutic tool' [Chan].

In assessing clients' conditions, standardized assessments such as goniometer, dynamometer, pinch gauge and sensibility tests were used to identify components' dysfunctions. Once the dysfunctions were identified, these occupation or purposeful tasks are mainly targeted to reduce the dysfunctions. For instance, "After I identified the problem through standardized assessments, I will choose the occupational tasks that are rich with therapeutic benefits to improve the 
OCCUPATION BASED INTERVENTION IN HAND INJURY REHABILITATION

components' functions" [Kesuma]. Participants regularly monitor their client's progression to make sure the occupational tasks prescribed were suitable and benefited the clients.

\section{Occupation as an end}

This theme describes how the occupational therapists provide 'occupation as an end'. Four subordinate themes emerged; evaluation of occupational performance, collaborative goal between therapist and client, direct occupational performance training and applying a continuum of treatment. Participants initiated 'occupation as an end' with an assessment to identify occupational performance problems. A complete occupational profile is obtained during the first session with the client. Observation, standardized and non-standardized assessments are often used in the evaluation process. "We [occupational therapists] use Modified Barthel Index (MBI) as stated in the Standard Operating Procedure of Malaysian Occupational Therapy Service" [Nurul]. Most participants described that MBI as a screening tool is not sensitive enough to detect occupational performance issues of their clients with hand injuries. Clients were counter assessed with the Canadian Occupational Performance Measure (COPM) or other functional assessments such as Functional Independence Measure (FIM), Disability of Arm, Shoulder and Hand (DASH) and Jebsen-Taylor Hand Function Test (JHFT). Participants use a top-down approach for assessing clients, where assessments begin with occupational performance issues and then moves down to components' dysfunctions.

Goals were set collaboratively with the clients when the occupational performance problems were identified. Participants discuss and design the intervention plan according to the client's priorities and needs. "I will discuss with clients about their occupational performance problems, 
and then decide which problems should be intervened in the first place" [Anita]. COPM was mentioned by most participants to identify the clients' priorities and occupational performance problems.

Participants described 'occupation as an end' in two ways; direct occupational performance training or using a continuum of treatment. A direct occupational training uses at the initial stage of injury, which involves strategies such as providing aids, adaptations and environmental modifications to enable the clients to engage in their daily occupations. Participants explained the purpose of training to their clients and how they can perform the problematic occupations using these strategies that might also include a demonstration and practice where clients try to perform the tasks during the therapy session. Participants stated that they usually use whatever abilities and assets the clients have before they decided to provide assistive devices or do modifications. Zul said that, "Providing an assistive device is the last resort when the clients really need it".

Another way of providing 'occupation as an end' was through a continuum of treatment. Participants who described 'occupation as an end' involved various intervention types in occupational therapy as long as the end goal is occupational performance. "I use various methods to prepare the clients for occupational performance." [Daisy]. Participants highlighted adjunctive methods (e.g. exercises, splinting, physical agent modalities, and sensory stimulations etc.); enabling activities (e.g. simulated activities such as practice board, theraputty putty, fine motor activities etc.); and purposeful tasks (e.g. practice writing and typing, meal preparation tasks, practice dressing in clinic etc.) are the stepping stones for successful occupational 
OCCUPATION BASED INTERVENTION IN HAND INJURY REHABILITATION

performance. Implementing 'occupation as an end' using this approach empowers the clients to perform their occupations similar to before their injuries and is used when the clients have been trained and have mastered the direct occupational performance training.

\title{
Benefit of OBI
}

The benefit of OBI according to the following subordinate themes: enjoyable rehabilitation experience, one treatment with double benefits, identity of occupational therapy, cost effective and improve clients' satisfaction. OBI provides an enjoyable rehabilitation experience to both occupational therapists and their clients. Therapeutic relationships between clients and therapists are developed during the therapy session, which assists clients to feel more comfortable to share their problems and concerns. Most participants felt that OBI was more fun than doing hand exercises. "Engaging clients in their occupational tasks, either as a means or as an end is exciting rather than pressing theraputty putty" [Chan]. Another participant said "When using OBI as a means with traumatic hand injury clients, it could divert the clients' attention from pain and uneasiness of the injured hand" [Rosni].

\author{
Most participants described OBI as a treatment with double benefits. "Using OBI is like killing \\ two birds with one stone [double benefits]. It helps to improve occupational performance and \\ the function of the injured hand" [Billy]. All participants expressed that occupational therapists \\ should focus on OBI because it better represents the identity of profession. "Occupation is our \\ core treatment. It represents the identity of our profession and we should integrate the \\ occupation in hand therapy in a creative way" [Idris].
}


OBI was described as a cost effective treatment, where it can be used in the clinic and home as therapy modalities. OBI uses material and resources that are related to client occupations and their context (e.g. home, workplace and school). "I can open a hand therapy clinic without buying expensive equipment. I can turn the rubbish things such as rubber band and recycle springs into therapeutic tools ((Smile))" [Chan]. OBI was described to be helpful in improving client satisfaction. Some participants expressed that their clients felt surprised with the outcomes of OBI. "One of my clients said: I didn't know these simple tasks could make my hand better." [Kesuma].

\section{Challenges of OBI}

Several challenges of using OBI in hand injury rehabilitation arose. Five subordinate themes emerged; occupational therapist factors, client factors, credibility of occupation as treatment modalities, logistic factors and contextual factors. Occupational therapists were identified as one of the challenges as some participants felt that they were lacking creative skills to implement OBI. When being asked further about the creative skills, a participant answered "I've to be creative in selecting occupation as a remediation agent and I've to analyse the therapeutic benefits of the occupation." [Damia]. Some participants felt that their knowledge and training of OBI were lacking. "I was trained 18 years ago and at that time, there were no occupational frameworks or models. We [occupational therapist students] were trained based on the medical theories" [Bavani]. Bolhan said, "We [occupational therapists] claimed that we are expert in providing occupation, but we didn't have much knowledge and training about it'. Participants also described that the influence of colleagues was also a challenge to practise OBI. "I eagerly 
OCCUPATION BASED INTERVENTION IN HAND INJURY REHABILITATION

want to focus on occupation when I started my career, but I feel awkward when I'm the only one who practises this way" [Hussain].

Client factors were also a challenge. Most participants described that their clients were much more concerned with the symptoms and impairments rather than functions. Attention on reducing and eliminating impairments means occupational performance is disregarded. Some participants also worried about the clients' perception of occupational therapy intervention. "The clients might not have confidence with the intervention when we only use everyday occupations as therapy" [Bavani]. Some clients prefer high technology and sophisticated machineries rather than everyday occupations as therapy tools. As Nurul said, "I think clients are more confidence with the machines and equipment because they have no idea about the therapeutic benefits of occupation". Participants also described that their clients prefer hands-on therapy such as Range of Motion (ROM) exercise, massage and application of Physical Agent Modalities (PAM) than actively 'doing' occupational tasks during the therapy sessions.

Another challenge to OBI was the credibility of using occupation as the remediation agent. When being asked about 'occupation as a means', Bolhan said, "I rarely found evidence to support this practice". "This [occupation as a means] is such an old school and not straightforward treatment" [Bavani]. Participants also described that a strict therapy protocols such as for fracture, tendon and nerve repair restricts them from using OBI in their practice. For example, "I must follow the protocol to prevent tendon rupture during the protective stage and I wouldn't allow the clients to use their hand actively. The right time use it is when the client is in active rehabilitation phase" [Chan]. 
OCCUPATION BASED INTERVENTION IN HAND INJURY REHABILITATION

All participants expressed that logistics such as time, workload, space and resources are the barriers to practise OBI. "Providing occupation as an end requires one to one sessions and consumes so much time. It could not be done in a group as clients may have different occupational performance problems" [Kesuma]. Melur said that "Sometimes I need to attend to more than three clients at one time. I have to maintain my statistics as urged by the top management”. Some units were not set-up and equipped with appropriate facilities for OBI. "I've no space to do ADLs training or work rehabilitation program here. This unit is just equipped with modalities to treat impairments and body functions". Participants also described that resources for OBI in the unit are limited to basic ADLs only. Melati said that, "For IADLs, I don't usually train the client, but I do a purposeful task in the clinic and encourage the client to transfer the skills at home".

Other challenges to practise OBI in hand injury rehabilitation include contextual factors such as organizational culture, inadequate information for occupational therapy roles, no support from the top management of the organization, and the Malaysian culture of serving 'sick clients'. Participants felt that they have to follow what referring doctors order them to do as the doctors' position was perceived to be higher than the occupational therapists. "I've got to follow what the doctors order me to do as stated in the referral form" [Nurul]. Another participant said, "It's a dilemma whether to serve what the doctors ordered us to do or what occupational therapists actually should do" [Melati]. Participants also felt that referring doctors might not have any idea of what occupational therapist can offer, "Some doctors don't know what occupational therapists can do for hand clients." [Nurul]. Participants highlighted that they have no support from the top 
OCCUPATION BASED INTERVENTION IN HAND INJURY REHABILITATION

management to implement OBI. "Well, they just concern with the number of clients that we treat. It's difficult to get budget and new staff' [Kesuma]. OBI was difficult to implement within Malaysian culture, which supports the clients to take a sick role. "Sometimes, they [family members] are over protective to the clients, they still want to help even the clients can do by themselves" [Bavani].

\section{Making OBI a reality}

The last theme discusses several strategies to make OBI a reality in hand injury rehabilitation. Three subordinate themes emerged; education and information to all parties, considering the clients' occupational profile, and enhance the credibility of occupational therapists. Education and information to clients, caregivers, multidisciplinary professionals and all people who are involved in the rehabilitation process was highlighted as the most important things. "Clients actually have no idea what occupational therapy can offer them. They only expect medications and exercises when they came to the hospital." [Hussain]. Participants believed that if the information and education were provided diligently, the clients and caregivers would feel confident and more susceptible towards OBI. Participants stressed that sufficient and clear information regarding the role of occupational therapy should be delivered to multidisciplinary professionals through programs such as presentations, campaigns, back door promotions and hospital conferences.

Participants stressed the clients' occupational profile is necessary to provide the appropriate treatment for the clients. "Occupation as a mean and as an end must be relevant and related to the clients' daily living patterns, except the clients want to explore new things such as new 
OCCUPATION BASED INTERVENTION IN HAND INJURY REHABILITATION

hobbies, jobs and roles" [Chan]. Participant Shima provided an example "Let's say that the client is an assistant at a restaurant, domestic training is relevant and meaningful to him".

Enhancing the credibility of occupational therapists was one of the strategies suggested by the participants. Interpersonal and creativity skills of occupational therapists should strengthen OBI as a reality. "Creativity skill is important to design OBI while interpersonal skills are important to deliver OBI" [Chan]. "Occupational therapists should be more innovative in using occupations as the therapy agent, as well as setting occupation as an outcome of intervention" [Idris]. Participants felt that occupational therapists should be motivated, dynamic and cannot just rely on the knowledge from their pre-service training. Participants suggested that occupational therapists need to attend training, workshops and seminars to improve their knowledge and skills. Most participants agreed that OBI should be taught and emphasized since pre-service training. "I think OBI should be emphasized since the college or university time. The situation will be gradually changed if the occupational therapy students are trained with OBI.

[Hussain]. Finally, participants suggested occupational therapists are encouraged to use a reflective practice. "We can use the opportunity to overcome challenges and use strength to compensate weaknesses. Reflective practice is important to overcome all barriers to practise $O B \Gamma^{\prime}[$ Billy].

\section{Discussion}

This study describes the experiences of occupational therapists applying OBI using occupation as a means and an end. Findings from this study support previous evidence that occupation as a means and an end can be provided as a single therapy and can improve both occupational 
OCCUPATION BASED INTERVENTION IN HAND INJURY REHABILITATION

performance and function of injured hands (9). OBI is an individualized and a client-centred intervention $(8,13,16)$. Occupation as a means (bottom-up) and an end (top-down) use different evaluation approaches. Regardless of the approaches used, choosing sensitive and reliable tools is important as occupational performance is difficult to quantify (28). Although occupation as a means and an end has different purposes, the ultimate goal is the same: to promote independence and enhance quality of life of the clients.

Findings from this study further echo the notion of occupation as a means and an end.

'Occupation as a means' aims to rehabilitate impaired physical function using occupational or purposeful tasks $(9,10)$. For the best outcome, the therapeutic power of the occupational task must be analysed before it is prescribed to the client $(9,10)$. Although 'occupation as a means' can be a simple behavior or equal to purposeful tasks, it must be related to clients' daily occupations to motivate and help them to transfer the skills for occupational performance (9). The tasks for 'occupation as a means' can be graded to suit the clients' abilities to avoid frustration and should be more challenging than what the clients can currently do for the prospect of success (10). The clients are encouraged to use the injured hand as much as possible when doing occupational tasks to improve hand functions and to avoid 'learned non-use' (19).

\footnotetext{
'Occupation as an end' that aims to improve a client's occupational performance either using direct occupational performance training or a continuum of treatment. Direct training occupational performance training aims to help the clients to cope with restricted daily living activities at the initial stage of hand injury, while a continuum of treatment aims to enable the clients to perform daily occupations as well as before the injuries. Direct training uses whatever
} 
abilities and assets the clients have, and may involve adaptation strategies, aids and environmental modifications $(1,10)$. A continuum of treatment involves preparatory methods (adjunctive and enabling activities) and purposeful tasks to prepare the clients for occupational performance (9). When using this approach, occupational therapists must be able to link and direct the treatment continuum towards occupational performance goals $(12,29)$.

Providing OBI is strongly influenced by the concept and foundation of the occupational therapy profession, which is to place occupation at the centre of practice (12). In this study, occupational therapists emphasized that OBI represents the professional identity and makes their practice unique $(12,15,16)$. OBI is more enjoyable, helps to develop therapeutic relationship between therapist and clients, can divert clients' attention from the painful traumatic hand and helps to improve their treatment satisfaction $(16,19,20)$. However, logistic issues such as time, workload and the clinic environment are the barriers to the implementation of OBI in hand injury rehabilitation $(16,20,28,30)$. The clients who are concerned about their impairment and symptoms, prefer high technology and hands-on exercise, and assume that OBI is not important, and that assumption also remains as a barrier to apply OBI (20). Besides, occupational therapists in Malaysia also perceive that $\mathrm{OBI}$ is more difficult compared to component-driven interventions (31), occupational therapists find there is limited evidence on the efficacy of OBI for clients with hand injuries $(9,20)$. This study highlights some contextual barriers such as the bureaucratic culture within Ministry of Health Malaysia and the Malaysian culture of serving sick clients as the challenges to implement OBI. Similarly with other Asian cultures, Malaysian culture permits regression and dependency when health events happened to family members 


\section{Implication for practice and research}

The findings of this study suggest several strategies to overcome the challenges in implementing OBI. First, the clients, caregivers and multidisciplinary members should be provided with adequate information and education about the purpose of OBI (20). Second, formal and informal training are needed to improve knowledge and skills of the occupational therapist in practising OBI. Occupational therapy schools should update the syllabus and curriculum, and equip the students with current knowledge and evidence around occupational therapy models and occupational sciences $(30,33)$. Third, occupational therapists should be reflective practitioners to overcome the barriers of OBI. Fisher stresses that occupational therapists must be able to reflect on what they do, how they do and what they think about their own doing to be more occupationbased practitioners (12).

The need for more evidence of occupation as a means and an end to support practice is highlighted in this study. More research on the effectiveness OBI in improving impaired functions and occupational performance for the clients with hand injuries are needed. Future research is also need to focus on exploring OBI in other areas of practice because the way OBI is practised and challenged may be different. Occupational therapists is suggested to establish OBI in every area to enhance occupation based practice, which directly connects the core tenets of the profession (34).

\section{Limitations}


One limitation of the study was that all participants were recruited from the Ministry of Health Malaysia hospitals, and the findings might not reflect the experience of those who work in private and independent centres. Another shortcoming was the member checking method used to improve the trustworthiness of study, where only four participants volunteered to participate in the focus group discussion. The terms 'occupation as a means and an end' were new to some participants, and might restrict the participants from giving good responses. Finally, the results of this qualitative study may not be generalizable to broader populations.

\section{Conclusion}

This study presents the experiences of occupational therapists in placing occupation at the centre of practice in an area that is dominated by the medical-oriented model. The perceived benefits, challenges and the struggles of the occupational therapists in providing OBI for the clients with hand injuries are highlighted. This study provides some insights and understandings for using occupation optimally in practice through the concept of occupation as a means and an end. OBI articulates well with the holistic concept of occupational therapy, where occupation can be used as the agent to reduce impairments, and help to improve clients' functional status and ability to engage in meaningful occupations.

\section{Acknowledgement}

The authors would like to thank all participants, the head of Malaysia Occupational Therapy Service and Malaysian Occupational Therapy Association. This study was supported by funding from the College of Healthcare Sciences, Division of Tropical Health and Medicine, James Cook University. 
OCCUPATION BASED INTERVENTION IN HAND INJURY REHABILITATION

\section{References}

1. American Occupational Therapy Association. OCCUPATIONAL THERAPY PRACTICE FRAMEWORK: Domain \& Process, 3rd Edition. American Journal of Occupational Therapy. 2014:S1-s48.

2. Fitzpatrick N. A phenomenological investigation of the experience of patients during a rehabilitation programme following a flexor tendon injury to their hand. British Journal of Hand Therapy. 2007;12(3):76-82.

3. Kingston G, Tanner B, Gray MA. The functional impact of a traumatic hand injury on people who live in rural and remote locations. Disability \& Rehabilitation. 2010;32(4):326-35.

4. Abdullah S, Jaafar JM, Das S, Sapuan J. An insight into industrial accidents involving the hand. Clin Ter. 2009;160(6):427-33.

5. Social Security Organisation. Annual report 2012. Kuala Lumpur Social Security Organisation, 2012.

6. Shalimar A, Jamari S, Abdul Halim A, Roohi S, Naicker A. A 2- year demographig-study of domestic peadiatric hand injuries requiring surgical intervention in an urban hospital.

Malaysian Orthopaedic Journal. 2007;1(1):34-8.

7. Isa AR, Moe H. Fireworks related injuries during hari raya festival in Hospital Universiti Sains Malaysia. Medical Journal Malaysia. 1991;46(4):333-7.

8. Bear-Lehman J, Flinn-Wagner S. Hand rehabilitation and occupational tyherapy. Occupational Therapy in Health Care. 1988;4(3-4):7-15.

9. Gray JM. Putting Occupation into Practice: Occupation as Ends, Occupation as Means. American Journal of Occupational Therapy. 1998;52(5):354-64.

10. Trombly CA. Occupation: Purposefulness and Meaningfulness as Therapeutic Mechanisms. American Journal of Occupational Therapy. 1995;49(10):960-72.

11. Wilson L, Roden PW, Taylor Y, Marston L. The effectiveness of origami on overall hand function after injury: A pilot controlled trial. British Journal Of Hand Therapy. 2008;13(12-20).

12. Fisher AG. Occupation-centred, occupation-based, occupation-focused: Same, same or different? Scandinavian Journal of Occupational Therapy. 2013;20(3):162-73.

13. Pierce D. Occupation by design: Dimensions, therapeutic power, and creative process. American Journal of Occupational Therapy. 2001;55(3):249-59.

14. Golledge J. 'Is there unnecessary duplications of skills between occupational therapist and physiotherapist?'. British Journal of Occupational Therapy. 1998;61(4):161-2.

15. Burke JP. How therapists' conceptual perspectives influence early intervention evaluations. Scandinavian Journal of Occupational Therapy. 2001;8(1):49-61.

16. Estes J, Pierce DE. Pediatric therapists' perspectives on occupation-based practice. Scandinavian Journal of Occupational Therapy. 2012;19(1):17-25.

17. Schindler VP. A client-centred, occupation-based occupational therapy programme for adults with psychiatric diagnoses. Occupational Therapy International. 2010;17(3):105-12.

18. Zingmark M, Fisher AG, Rocklöv J, Nilsson I. Occupation-focused interventions for well older people: An exploratory randomized controlled trial. Scandinavian Journal of Occupational Therapy. 2014(0):1-11.

19. Earley D, Shannon M. The Use of occupational-based treatment with a person who has shoulder adhesive capsulitis: A case report. American Journal of Occupational Therapy. 2006;60(4):397-403. 
20. Colaianni D, Provident I. The benefits of and challenges to the use of occupation in hand therapy. Occupational Therapy in Health Care. 2010;24(2):130-46.

21. Rogers S. Occupation-based intervention in medical-based settings. OT Practice. 2007;12(15):10-6.

22. Smith JA. Beyond the divide between cognition and discourse: Using interpretative phenomenological analysis in health psychology. Psychology and Health. 1996;11:261-71.

23. Smith JA. Reflecting on the development of interpretative phenomenological analysis and its contribution to qualitative research in psychology. Qualitative Research in Psychology. 2004;1(1):39-54.

24. Fade S. Using interpretative phenomenological analysis for public health nutrition and dietatic research: A practical guide. Proceeding of the Nutrition Society. 2004;63:647-53.

25. Unsworth $\mathrm{C}$. The clinical reasoning of novice and expert occupational therapists Scandinavian Journal of Occupational Therapy. 2001;8:163-73.

26. Patton MQ. Qualitative Research \& Evaluation Methods. 3rd ed. Thousand Oaks: Sage; 2002.

27. Smith JA, Flowers P, Larkin M. Interpretative phenomenological analysis theory, method and research. Los Angeles: Sage 2009.

28. Chisholm D, Dolhi C, Schreiber J. Occupational therapy intervention resource manual. Australia: Thomson Delmar Learning; 2004.

29. Early MB. Occupational therapy and physical disabilities In: Early MB, editor. Physical Dysfunction Practice Skill for the occupational therapy assistant 3ed. United States: Elsevier; 2013. p. 2-16.

30. Stack R, Barker D. Students as advocates for occupation-based practice. Occupational Therapy Now. 2011;13(3):13-5.

31. Goldstein-Lohman H, Kratz A, Pierce D. A study of occupation-based practice. In: Pierce D, editor. Occupation by design building therapeutic power. Philadelphia: F.A. Davis Company; 2003. p. 239-61.

32. Nilchaikovit T, Hill JM, Holland JC. The effects of culture on illness behavior and medical care: Asian and American differences. General Hospital Psychiatry. 1993;15(1):41-50. 33. Whiteford GE, Wilcock AA. Centralizing occupation in occupational therapy curricula: Imperative of the new millennium. Occupational Therapy International. 2001;8(2):81-5. 34. Pierce D. How can the occupation base of occupational therapy be strengthened? Australian Occupational Therapy Journal. 2003;50(1):1-2. 
OCCUPATION BASED INTERVENTION IN HAND INJURY REHABILITATION

Table

\section{Legends of tables and figures}

Table 1. Participants' Descriptions (Age range: 30-51 years, Mean age: 40.81)

\begin{tabular}{|c|c|c|c|c|}
\hline Pseudonym & Gender & Qualification & $\begin{array}{c}\text { Experience } \\
\text { (years) }\end{array}$ & $\begin{array}{l}\text { Direct contact with } \\
\text { hand clients ( } \% \text { of } \\
\text { total client load per } \\
\text { month) }\end{array}$ \\
\hline Damia & Female & Dip. OT & 5 & 95 \\
\hline Billy & Male & Dip. OT & 7 & 100 \\
\hline Husain & Male & BSc. OT & 6 & 50 \\
\hline Idris & Male & BSc. OT & 6 & 60 \\
\hline Melur & Female & BSc. OT & 6 & 80 \\
\hline Daisy & Female & Dip. OT & 7 & 100 \\
\hline Anita & Female & BSc. OT & 8 & 70 \\
\hline Rosni & Female & Dip. OT & 7 & 90 \\
\hline Melati & Female & BSc. OT & 6 & 95 \\
\hline Bavani & Female & BSc. OT & 7 & 80 \\
\hline Kesuma & Female & Dip. OT & 5 & 100 \\
\hline Chan & Male & BSc. OT & 18 & 40 \\
\hline Bolhan & Male & BSc. OT & 6 & 90 \\
\hline Nurul & Female & Dip. OT & 5 & 90 \\
\hline $\mathrm{Zul}$ & Male & Dip. OT & 5 & 95 \\
\hline Shima & Female & Dip. OT & 6 & 70 \\
\hline
\end{tabular}

